(C) 2005 The Japan Society of Applied Physics

\title{
Electric Field Imaging Using Intense Half-Cycle Terahertz Pulses
}

\author{
Rakchanok RungSAWANG*, Keiji TuKAmOTO and Toshiaki HATTORI \\ Institute of Applied Physics, University of Tsukuba, 1-1-1 Tennodai, Tsukuba 305-8573, Japan
}

(Received July 17, 2004; accepted December 15, 2004; published April 8, 2005)

Characteristics of terahertz $(\mathrm{THz})$ imaging using approximately half-cycle $\mathrm{THz}$ pulses were studied using a large-aperture biased photoconductive antenna which emits an intense $\mathrm{THz}$ field with a large $\mathrm{THz}$ beam. Studies of the dependence of the $\mathrm{THz}$ image quality on delay time show that real-time imaging is possible if the probe pulse is timed appropriately. The best images were observed at a time window when the THz field had a steep transient. This allows two-dimensional imaging in a fixed-time mode, which enables observation of a nonstationary object. Using phase-sensitive electrooptic detection and scanning a delay time, not only two-dimensional time-domain spectroscopy is possible, but frequency-resolved images are also available. Depending on the frequency, we found that images of the actual shape or the edge of an object were obtained. [DOI: 10.1143/JJAP.44.1771]

KEYWORDS: terahertz imaging, half-cycle electric pulse, photoconductive antenna, phase-sensitive detection, frequencyresolved image

\section{Introduction}

The advantage of terahertz ( $\mathrm{THz}$ ) electromagnetic pulses is that they are noninvasive and phase information can be obtained. Because of this, they are used in imaging and have recently attracted a large amount of interest. THz imaging was introduced by $\mathrm{Hu}$ and Nuss ${ }^{1)}$ in 1995 . Usually when obtaining images using $\mathrm{THz}$ pulses, temporal waveforms are measured at all image positions. Two-dimensional (2D) images can then be reconstructed from these data. ${ }^{1-4)}$ The drawback of this technique is that it is usually necessary to scan the beam over the object of interest. The speed of the optical delay line and the time required to move the sample from point to point limit the acquisition time. One can reduce the acquisition time by capturing the $2 \mathrm{D}$ image at a fixed time delay using a charge-coupled device (CCD) camera. Wu et al. first obtained a 2D field profile of a $\mathrm{THz}$ beam using an electrooptic (EO) method and a CCD camera. ${ }^{5)}$

An important factor in achieving 2D simultaneous imaging is the power of the $\mathrm{THz}$ source, especially in a transmission mode. An intense $\mathrm{THz}$ field plays an important role in real-time $\mathrm{THz}$ detection. In addition, as the $\mathrm{THz}$ field intensity increases, a thicker sample or a higher density of moisture can be penetrated. High power coherent $\mathrm{THz}$ radiation is available from several sources such as biased photoconductive antennas, ${ }^{6,7)}$ phase-matched difference frequency mixing in a GaSe crystal, ${ }^{8)}$ and relativistic electrons. ${ }^{9)}$ Of these sources, a biased photoconductive antenna is the most compact. It gives an electric field pulse of a halfcycle to nearly a monocycle that has been used in timedomain spectroscopies and studies of Rydberg atom ionization. ${ }^{10)}$ The advantage of using half-cycle pulses in 2D imaging is that the electric field has a well-defined field distribution in the spatial and time domains. ${ }^{11)}$ Usami et al. used a biased large-aperture antenna in performing 2D imaging by fixing the time delay between the THz pulse and the probe pulse. ${ }^{12)}$ Optimization of the time delay to obtain the best image quality, however, has not been investigated. Optimized timing is an importance factor in a fixed-time delay mode. The advantage of this mode is that enables in situ studies of dynamic processes or high-speed single-time

*E-mail address: nok@laserlab.bk.tsukuba.ac.jp events at a high frame rate. ${ }^{13,14)}$

Frequency-resolved images can in general yield better resolution, although their acquisition time should be much longer than that for fixed-time images. By scanning the delay time and Fourier transforming the temporal waveforms of the $\mathrm{THz}$ field at each position, frequency-resolved images can be obtained. Use of real-time acquisition with a CCD camera reduces the overall time required for the measurements.

In this study, we describe the results of two experiments concerning the use of intense half-cycle $\mathrm{THz}$ pulses from a large-aperture antenna in imaging: delay-time optimization and frequency-resolved imaging. We used the phase-sensitive electrooptic detection method ${ }^{15,16)}$ and imaged a metal object in a transmission mode. Digital processing was introduced to eliminate the artifacts from a nonuniform $\mathrm{THz}$ beam profile.

\section{THz Field Imaging System}

Figure 1 illustrates the experimental setup, which is similar to that described in a previous paper. ${ }^{11)}$ The optical source was a regeneratively amplified Ti: sapphire laser with a $150 \mathrm{fs}$ pulse width, $800 \mathrm{~nm}$ central wavelength, and a $1 \mathrm{kHz}$ repetition rate. The laser beam was divided into a major intensity portion for the pump beam and a minor portion for the probe beam. The pump beam was spatially expanded and illuminated a large-aperture photoconductive antenna, com-

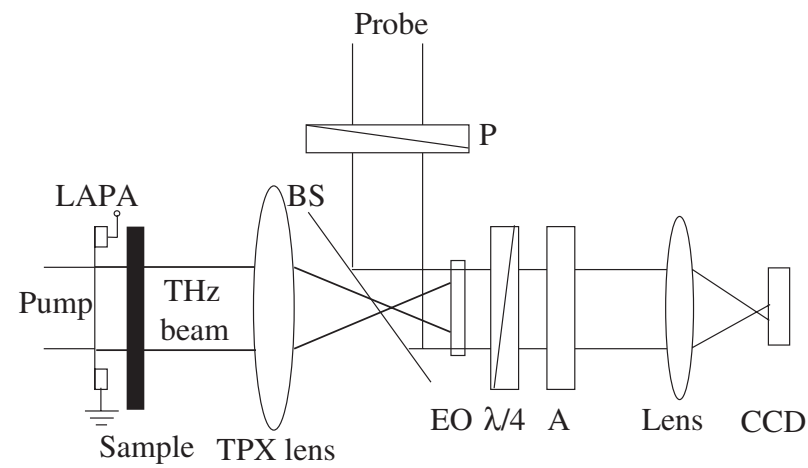

Fig. 1. Schematic of the experimental set up. LAPA: large-aperture photoconductive antenna, BS: pellicle beam splitter, EO: electrooptic crystal $(\mathrm{ZnTe}), \lambda / 4$ : quarter-wave plate, $\mathrm{P}$ : polarizer, A: polarization analyzer. 
posed of a semi-insulating GaAs wafer and two aluminum electrodes with a $3-\mathrm{cm}$ intergap spacing. A pulsed high bias voltage was applied to the wafer through the electrodes. The pump pulses created carriers in the intergap area, leading to the generation of $\mathrm{THz}$ radiation. In this study, the pump pulse energy and the bias voltage were $500 \mu \mathrm{J}$ and $5 \mathrm{kV}$, respectively. The THz beam emitted from the photoconductive antenna passed through a sample object and a lens made of polymethylpentene (TPX; Mitsui Chemicals, Inc.). The TPX lens and an EO crystal were placed so that the $3: 2$ image of the object was formed on the EO crystal. The focal length of the lens was $f=98.3 \mathrm{~mm}$ and its diameter was $80 \mathrm{~mm}$. The object and its image position were set at $z=$ $-5 f / 2$ and $z=5 f / 3$ when the lens was placed at $z=0$.

The spatial distribution of the refractive index modulation in the EO crystal, which is proportional to the $\mathrm{THz}$ electric field, was mapped by the probe optical pulses onto a CCD camera. By generating a local oscillator field using a quarterwave plate, we detected the $2 \mathrm{D}$ field image with high sensitivity. The probe beam went through a delay line and was linearly polarized in the horizontal direction. The beam was then expanded and led to the EO crystal by a pellicle beam splitter. A 1-mm-thick $\langle 110\rangle$ ZnTe crystal with an area of $18 \times 20 \mathrm{~mm}^{2}$ was used as the EO crystal. The optical component placed immediately after the EO crystal was different from that used in the conventional $\mathrm{THz}$ intensity detection method. ${ }^{11)}$ In the conventional method, the EO crystal is followed by a polarization analyzer that is oriented perpendicularly to the polarizer orientation. In this study, a quarter-wave plate was additionally inserted between the EO crystal and the crossed analyzer for the electric field detection. The axis of the quarter-wave plate was adjusted to be parallel to the axis of the polarizer. The analyzer was then rotated with a small angle, $\delta$, in order to induce a local oscillator field, which is a phase-shifted fraction of the probe light. This method is called optical heterodyne detection (OHD). ${ }^{16,17)}$ The phase retardation angle of the probe light due to the EO effect, $\theta$, which is proportional to the $\mathrm{THz}$ electric field, is given by (also in appendix) $)^{15}$ )

$$
\theta=-\delta+\sqrt{\delta^{2}+\frac{I-I_{\mathrm{b}}}{I_{0}}},
$$

when $|\delta|$ and $|\theta| \ll 1$. Here, $I_{0}$ is the incident probe light intensity, $I_{\mathrm{b}}$ is the background intensity of the probe light transmitted through the $\delta$-rotated analyzer, and $I$ is the intensity of the transmitted probe light in the presence of the $\mathrm{THz}$ field. It should be noted here that $I_{\mathrm{b}}$ is contributed to by optical bias light from the rotation of the analyzer axis by $\delta$ and also by the intrinsic birefringence and light scattering in the EO crystal. In our experiment, the orientation angle of the analyzer was set at $\delta=0.02$ radians, at which probe intensity modulation due to the EO effect was almost optimized. ${ }^{16)}$ From eq. (1), we see that both positive and negative electric fields can be detected depending on the relation between $I$ and $I_{\mathrm{b}}$. The sensitivity of this detection method is better than that of the conventional intensity detection method. The largest magnitude of the negative value of $\theta$ detectable with this method is $-\delta$ as can be seen from eq. (1). If larger phase modulation is expected, the orientation angle, $\delta$, must be set at a larger value or a negative value. The spatial distribution of the probe light intensity on the EO crystal was imaged onto the CCD device using an optical lens by reducing the image size by a factor of 5. The exposure time of the camera was set to $30 \mathrm{~ms}$, which corresponds to the accumulation of 30 shots of $\mathrm{THz}$ pulses for a single image.

\section{Time-Resolved THz Field Images}

Spatial and temporal profiles of $\mathrm{THz}$ pulses depend very much on the propagating and observing conditions because the bulk of their energy is composed of low-frequency components. To obtain good image quality in $\mathrm{THz}$ real-time imaging with a fixed time delay, the dependence of the image quality on the delay time must be understood. In this section, we describe the relationship between the image quality and the on-axis $\mathrm{THz}$ waveform.

A previous paper described how the waveform of halfcycle $\mathrm{THz}$ pulses changes during its propagation in free space and during focusing. ${ }^{18)}$ In this experimental setup, the $\mathrm{THz}$ wave emitted from the large-aperture antenna behaves like a plane wave and is focused at the focal point of the TPX lens in the absence of the sample. The temporal waveform of the $\mathrm{THz}$ pulse at the focus point is expected to be almost a half-cycle. Since the observation plane is far from the focus point, a waveform similar to the time integral of the half-cycle spectral shape is expected to be observed resulting from diffraction of high-frequency components. This occurs because the spectral filtering is inversely proportional to the frequency. We measured the on-axis temporal $\mathrm{THz}$ waveforms at $z=f$ and $z=5 f / 3$ without sample objects using the conventional balanced detection method. The distance between the antenna and the TPX lens was retained as in the imaging experiment, i.e., $300 \mathrm{~mm}$. Figure 2 shows the waveforms obtained. Since the measurements were performed separately from the imaging experiments, the values of the delay times do not correspond to those of the image data. The waveform at the focus point was found to be almost a half-cycle, having a tail with a small negative value. The existence of the negative tail shows that low-frequency components are diffracted out of the beam path before being focused while high-frequency components are not. By placing the lens closer to the $\mathrm{THz}$ source, a cleaner half-cycle waveform was observed at the

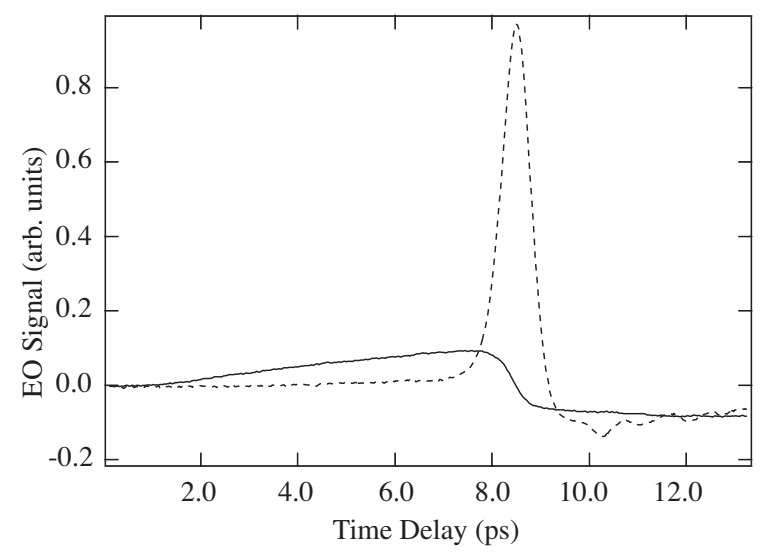

Fig. 2. On-axis temporal waveforms on the focal plane, $z=f$, (dotted line) and on the image plane, $z=5 / 3 f$, (solid line) measured using the conventional balanced detection method. 
focus point. The waveform observed on the image plane is composed of a slow (about $6 \mathrm{ps}$ ) rise, a fast decrease, and a negative tail which is almost constant in the observation time window. Imaging measurements were carried out in the delay time region between 2 and $10 \mathrm{ps,} \mathrm{which} \mathrm{roughly}$ corresponds to the delay time region from 0 to $8 \mathrm{ps}$ in the image data, where a significant temporal change in $\mathrm{THz}$ field is observed. The waveforms observed allowed us to understand the characteristics of $\mathrm{THz}$ radiation in this imaging system and under the temporal range of observation.

Using this $\mathrm{THz}$ pulse, we obtained time-resolved field images of an object. The sample object used was a metal rod concealed in a paper box. The diameter of the rod was $2 \mathrm{~mm}$. The box had dimensions of 6.8 (width) $\times 2.0$ (thickness) $\times$ 10.0 (height) cm, and the thickness of the paper was about $500 \mu \mathrm{m}$. This box was placed $10 \mathrm{~mm}$ from the $\mathrm{THz}$ emitter with the largest face parallel to the emitter surface. The metal rod was attached in the diagonal direction to the inside of the face of the box nearest to the $\mathrm{THz}$ antenna. Without the rod, the $\mathrm{THz}$ pulse waveform observed on the image plane using an EO sampling method underwent little change. Its shape or amplitude was not altered by the insertion of the paper box, and only a 1.17 ps delay in the peak arrival time was observed. Using the OHD method, we detected the 2D distribution in the intensity of the probe beam $I_{0}$, the background intensity $I_{\mathrm{b}}$ and the time-dependent signal intensity $I$. By substituting them into eq. (1), the THz field images were obtained. Figure 3 illustrates gray scale mappings of the electric field images obtained at several delay times without (reference images: left column) and with (sample images: right column) the metal rod in the box.

When the sample object was removed, the delay time dependence of the spatial distribution of the $\mathrm{THz}$ field behaved similarly to that reported in a previous paper, ${ }^{11)}$ as shown in the left column in Fig. 3. The image shown in Fig. 3(a) corresponds to the peak time of the $\mathrm{THz}$ pulse waveform at the center of the beam. In the time window shown here, a hole, the black area at the center of $\mathrm{THz}$ beam, appeared, as in Fig. 3(c), and became larger as the delay time increased, as in Fig. 3(e). The existence of a dark area at the right bottom corner in all images is attributed to an inhomogeneity of the pellicle beam splitter. The reflectivity of the beam splitter had a smaller value in this area. The ambiguous images may be caused by a low signal-to-noise ratio of approximately $16 \mathrm{~dB}$ and by the quality of the EO crystal. In Fig. 3(b), which is the image of the sample object obtained at the same delay time as that of Fig. 3(a), the shape of the rod is not observed clearly. At times later than the peak, as shown in Figs. 3(d) and 3(f), we can ascertain the rod as a dark area in the sample images, although its width in image (f) is much larger and the length is shorter than those of the actual object. These artifacts can be explained by the ring formation phenomena, i.e., focused half-cycle $\mathrm{THz}$ pulses form like an annual ring because of frequency-dependent focal beam sizes ${ }^{11)}$ and a major portion of long-wavelength components.

This experiment indicates that an appropriate time period exists for observing a clear image of an object. We extracted the temporal waveforms of the $\mathrm{THz}$ field at the center of the image from a series of images taken every $33 \mathrm{fs}$ of delay time. Figure 4 shows the waveforms observed with and
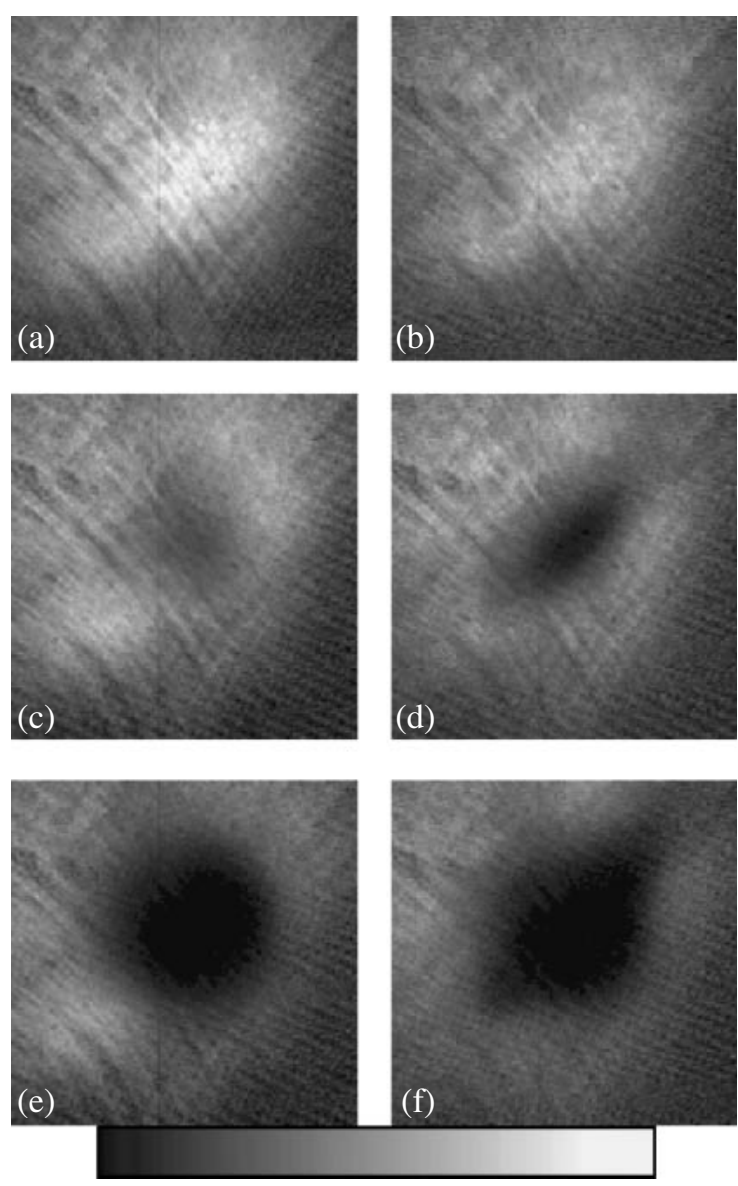

$-0.000984$

0.007063

Fig. 3. Left column: reference images; electric field distributions obtained using an empty paper box as an object. Right column: sample images; field distributions obtained with a metal rod in the paper box. Images in (a) and (b) were obtained at a delay time of $5.0 \mathrm{ps,} \mathrm{(c)} \mathrm{and} \mathrm{(d)} \mathrm{at} 5.7 \mathrm{ps}$, and (e) and (f) at $6.0 \mathrm{ps}$. These delay times correspond to those in Fig. 4. The color coding indicates the value of $\mathrm{THz}$-field-induced phase modulation, $\theta$, in eq. (1). Each image corresponds to an area of $27 \times$ $27 \mathrm{~mm}^{2}$ on the object plane.

without the metal rod in the paper box. The insert shows the time-derivative of the phase modulation. To obtain the timederivative curves, the original waveforms were smoothed by low-pass filtering below $4 \mathrm{THz}$ in the Fourier domain. Waveforms were also extracted at several off-axis points, although they are not shown here. The off-axis waveforms were similar to those observed on-axis with differences in arrival time and duration of the decrease. The appropriate time of 5.7-5.8 ps, which was used to observe the image in Fig. 3(d), corresponds to the time window where the temporal change in the $\mathrm{THz}$ field was largest (see peaks in the time-derivative of the phase modulation in the inset of Fig. 4). Interestingly, it was not the period where the difference between the two waveforms is largest, i.e., around 5 ps. This observation can be explained by considering the spectrum of the half-cycle pulse. Since the amplitude of lowfrequency components is relatively high, this contributes to the strength of the $\mathrm{THz}$ field, especially at the peak in the $\mathrm{THz}$ pulse. For this reason, we cannot observe the object at the peak time because the low-frequency components deteriorate the spatial resolution. In other words, the amplitude of the high-frequency components is largest at 


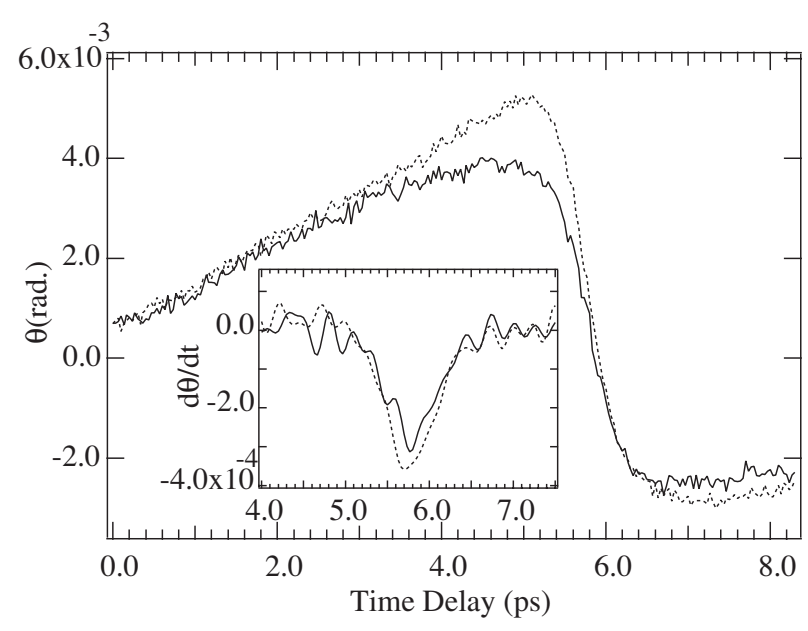

Fig. 4. The time-dependent phase modulation extracted from the central pixels of images in Fig. 3: reference (dotted line), sample (solid line). The dotted line shows the waveform observed using an empty paper box as an object, and the solid line shows that observed with a metal rod in the box. The inset shows the time-derivative of the phase modulation.

the delay time with the steepest transients in the THz field.

We introduced digital processing to eliminate the artifacts which result from the characteristics of the focused $\mathrm{THz}$ beam itself. This was accomplished by considering the difference and the ratio of sample images obtained by passing the $\mathrm{THz}$ beam through the rod inside the box with respect to the reference images without the rod at the same time delay. In the left column of Fig. 5, the difference between the images is shown. These were obtained by subtracting the reference image data from the sample image data at the corresponding time delay. Division images, which were obtained by dividing the sample image data by the reference image data, are shown in the right column of Fig. 5. With a time delay of $5.0 \mathrm{ps,} \mathrm{which} \mathrm{corresponds} \mathrm{to}$ peaks in the $\mathrm{THz}$ waveforms, the metal rod appears as a round dark area in the difference image shown in Fig. 5(a), while the rod in the division image at the same delay time, Fig. 5(b), is not clear. At this delay time, the difference in the field with and without the rod is large but the ratio is not, which can be verified by Fig. 4. Around the peak time, the temporal change in $\mathrm{THz}$ field is relatively small. The $\mathrm{THz}$ field around the peak time is contributed to mainly by lowfrequency components. This can explain the low spatial resolution observed in Fig. 3(d). The shape of the rod was best reproduced at $5.7 \mathrm{ps}$, both for the difference and division images, as shown in Figs. 5(c) and 5(d). The division image produced an object shadow closer to the actual sample shape than did the difference images. The smudge at the central area of images at $6.0 \mathrm{ps}$ shown in Figs. 5(e) and 5(f) is attributed to the characteristic of the focused $\mathrm{THz}$ beam itself as mentioned previously.

In summary, the dominance of low-frequency components of a half-cycle $\mathrm{THz}$ pulse does not allow the object to be visualized over all delay times. For a real-time imaging system, the time delay should be fixed at a time when the $\mathrm{THz}$ field changes rapidly with time. Image processing by dividing the sample image by the reference image gave the best result because of the absence of a $\mathrm{THz}$ beam profile effect, as seen in Fig. 5(d). However, this method limits some applications in which envelopes cannot be separated
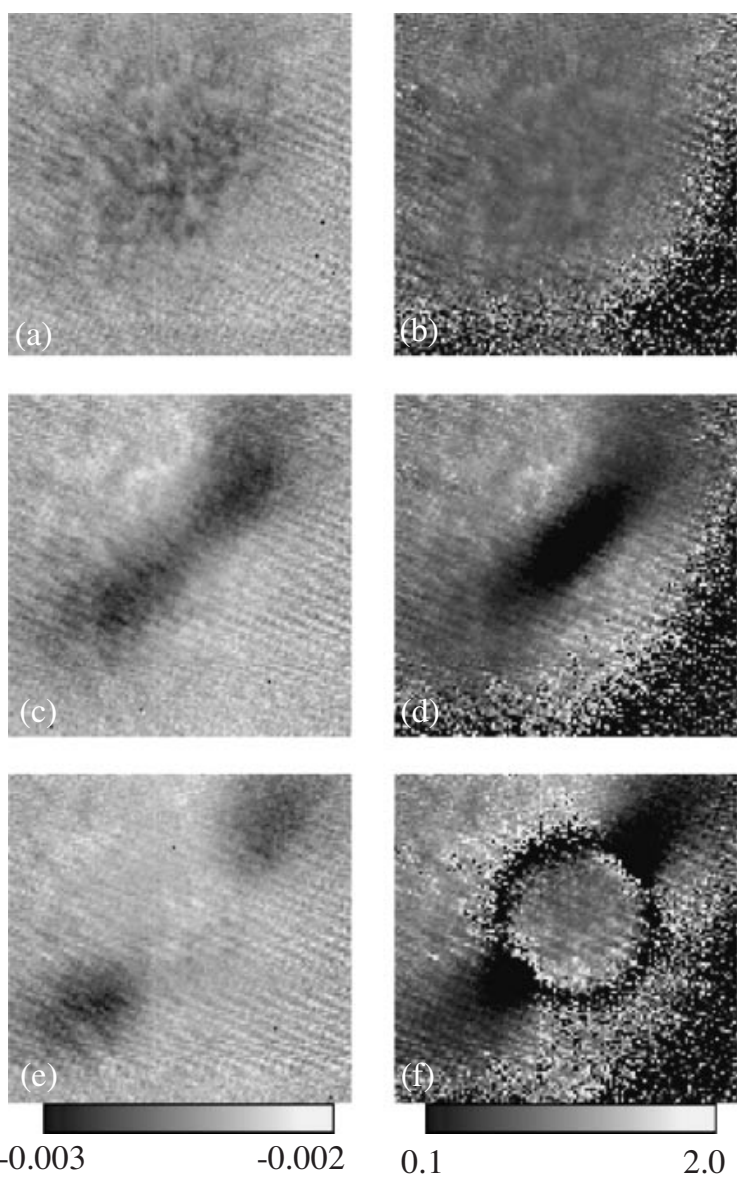

Fig. 5. Left column: difference images, obtained by subtracting from the sample image data in Fig. 3 the reference image data at respective delay times. Right column: division images, obtained by dividing the sample image data by the reference image data. Delay times at each line are the same as used in Fig. 3.

for recording the reference image. This problem can be solved if one knows the difference in delay times, $\Delta t$, when the $\mathrm{THz}$ pulse goes through the envelope and air. Assuming the envelope is homogeneous and that the $\mathrm{THz}$ beam profile is not changed after passage, the reference image corresponds to the air-passing $\mathrm{THz}$ image taken at a delay time advanced by $\Delta t$ with respect to the delay time of taking the sample image.

\section{Frequency-Resolved Amplitude Images}

Besides enabling weak $\mathrm{THz}$ signal detection, the OHD method has the advantage that field amplitude and relative phase at specific frequencies can be obtained by Fourier transformation. By scanning the optical delay between the pump and probe pulses, we obtained the $\mathrm{THz}$ field waveform at each position in the image simultaneously. All the waveforms were Fourier transformed to yield frequencyresolved $\mathrm{THz}$ images. The calculation of the Fourier transformation dealing with 200 frames of time-resolved data takes only a few minutes. Each frame was resolved to $123 \times 123$ pixels. In the calculation, the electric field images were first calculated from the observed data. Then the temporal waveform was extracted at each spatial point from a series of time-resolved images and padded with zeros to a length of 1024 before calculating one-dimensional Fourier transformation. The Fourier amplitude image at $0.35 \mathrm{THz}$ for 

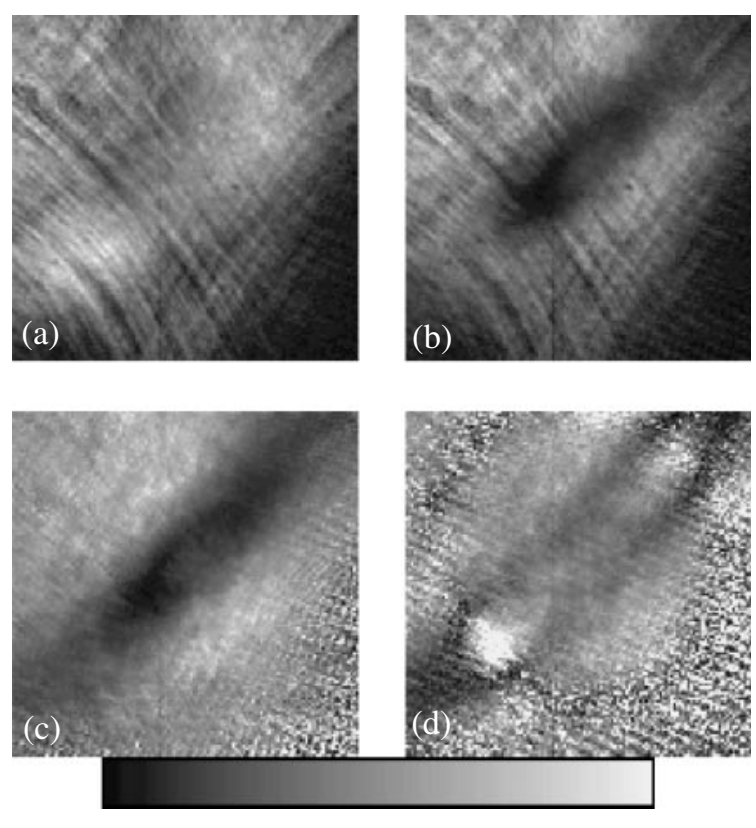

0.0

2.0

Fig. 6. Fourier amplitude images and division images. (a) is the Fourier amplitude image at $0.35 \mathrm{THz}$ calculated from the image series obtained using an empty box as the object and (b) is that obtained using a metal rod in the box. (c) is the division image at $0.35 \mathrm{THz}$, i.e., image (b) divided by image (a), and (d) is the division image at $0.53 \mathrm{THz}$.

the empty box used as the object and that of the metal rod in the box are shown in Figs. 6(a) and 6(b), respectively. When the frequency was scanned from low to high frequencies, the sample object first faintly appeared around $0.2 \mathrm{THz}$, which corresponds to the central frequency of the system bandwidth. Although the spectral image (b) shows the object's shape more clearly than the time-resolved image shown in Fig. 3(e), the whole rod is not revealed. This is attributed to the nonuniform spatial distribution of the $\mathrm{THz}$ field. $\mathrm{We}$ corrected for this effect by dividing image (b) by the reference image (a). The result is shown in Fig. 6(c). It can be seen clearly that the division image uncovered the entire shape of the sample in the observed area. Fig. 6(d) is the division image of the same sample at $0.53 \mathrm{THz}$. Interestingly, the dark area in this image corresponds to the boundary of the object. This can be attributed to the complex structure of a high-frequency amplitude profile of the $\mathrm{THz}$ pulse when the $\mathrm{THz}$ pulse passes through the lens, as observed by Matthew et al. ${ }^{19)}$

The following experiment was performed to observe other shapes in the frequency domain and to confirm the edge images at high frequencies that were found in the previous experiment. The samples were aluminum plates cut into triangular and circular shapes and attached to stiff paper. Each side of the triangle was $10 \mathrm{~mm}$ long and the diameter of the circle was $10 \mathrm{~mm}$. Figure 7 shows the Fourier amplitude images of the stiff paper (a)-(c), triangle (d)-(f) and circle (g)-(f) at frequencies $0.38 \mathrm{THz}$ (left column), $0.50 \mathrm{THz}$ (middle column) and $0.70 \mathrm{THz}$ (right column). The images at $0.38 \mathrm{THz}$ shown in Figs. $7(\mathrm{~d})$ and $7(\mathrm{~g})$ and those at $0.50 \mathrm{THz}$ shown in Figs. 7(e) and 7(h) reproduce the sample shapes as dark regions. On the other hand, the images at $0.70 \mathrm{THz}$ in Figs. 7(f) and 7(i) show the objects' boundaries as dark traces. In the reference image Fig. 7(c), the electric
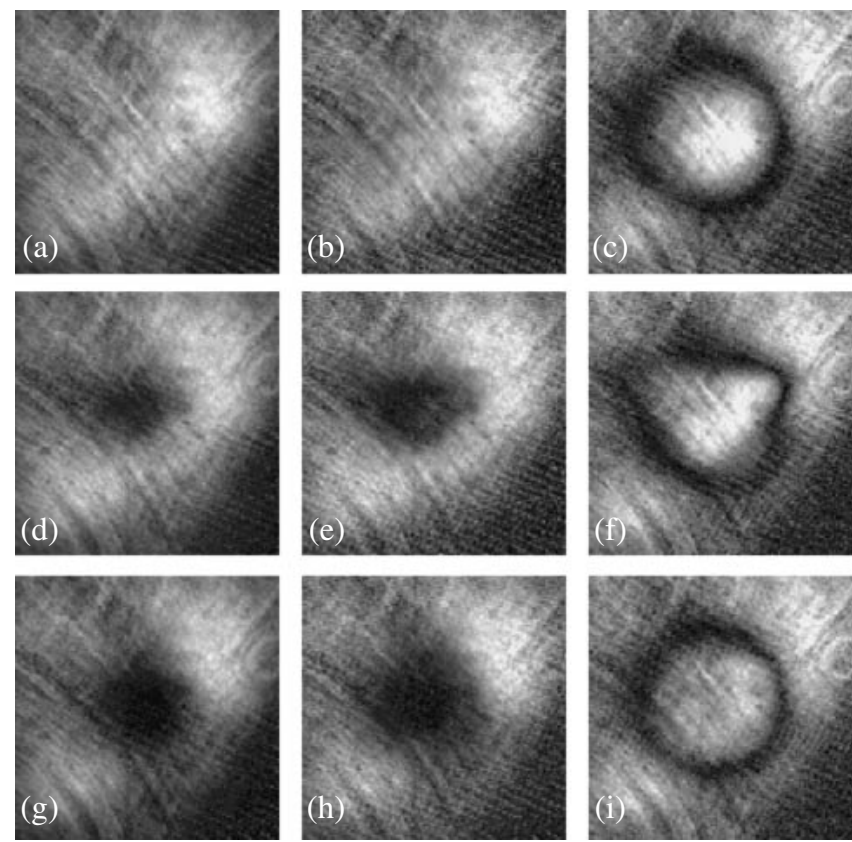

Fig. 7. Fourier amplitude images at frequencies $0.38 \mathrm{THz}$ (left column), $0.50 \mathrm{THz}$ (middle column) and $0.70 \mathrm{THz}$ (right column). Reference images (a)-(c) were obtained using stiff paper. The sets of sample images (d)-(f) and (g)-(h) were obtained using triangular and circular aluminum sheets attached to the stiff paper, respectively. Since the difference in signal amplitude among these frequency components is large, the same grey scale level is used only within each column.

field in the central part is low, as shown by the dark area. It can be presumed that the spatial field distribution of the $\mathrm{THz}$ beam is not Gaussian-like at some frequencies because of the spherical aberration of the thick TPX lens used in these experiments. This caused difficulties in identifying circular objects, as seen in Fig. 7(i). The image quality was improved by dividing the sample image by the reference image, although the result is not shown here.

\section{Conclusions}

Two dimensional $\mathrm{THz}$ imaging using half-cycle electromagnetic pulses from a large-aperture biased photoconductive antenna was performed. The best images of an object were obtained at a delay time with steep transients in the $\mathrm{THz}$ field. This feature indicates the possibility of real-time imaging by fixing the time delay at a time when a rapid change in THz field occurs when it is necessary to observe a moving object. The frequency-resolved images show the presence of an object from low frequency components while the images of their edges were found at comparatively high frequencies.

\section{Acknowledgements}

This study was partly supported by the 21 st Century Center of Excellence Program "Promotion of Creative Interdisciplinary Materials Science for Novel Functions".

1) B. B. Hu and M. C Nuss: Opt. Lett. 20 (1995) 1716.

2) M. C. Beard, G. M. Turner and C. A. Schmuttenmaer: Phys. Med. Biol. 47 (2002) 3841.

3) K. Kawase, Y. Ogawa, Y. Watanabe and H. Inoue: Opt. Express 11 (2003) 2549.

4) M. Herrmann, M. Tani and K. Sakai: Jpn. J. Appl. Phys. 39 (2000) 6254. 
5) Q. Wu, T. D. Hewitt and X.-C. Zhang: Appl. Phys. Lett. 69 (1996) 1026.

6) S.-G. Park, A. M. Weiner, M. R. Melloch, C. W. Siders, J. L. W. Siders and A. J. Taylor: IEEE J. Quantum Electron. 35 (1999) 1257.

7) H. Yoneda, K. Tokuyama, K. Ueda, H. Yamamoto and K. Baba: Appl. Opt. 40 (2001) 6733.

8) K. Reimann, R. P. Smith, A. M. Weiner, T. Elsaesser and M. Woerner: Opt. Lett. 28 (2003) 471.

9) G. L. Carr, M. C. Martin, W. R. McKinney, K. Jordan, G. R. Neil and G. P. Williams: Nature 420 (2002) 153.

10) C. Raman, C. W. S. Conover, C. I. Sukenik and P. H. Bucksbaum Phys. Rev. Lett. 76 (1996) 2436.

11) R. Rungsawang, K. Ohta, K. Tukamoto and T. Hattori: J. Phys. D 36 (2003) 229.

12) M. Usami, T. Iwamoto, R. Fukasawa, M. Tani, M. Watanabe and K. Sakai: Phys. Med. Biol. 47 (2002) 3749.

13) F. Miyamaru, T. Yonera, M. Tani and M. Hangyo: Jpn. J. Appl. Phys. 43 (2004) L489.

14) R. Rungsawasng, A. Mochiduki, S. Ookuma and T. Hattori: Jpn. J. Appl. Phys. 44 (2005) L288.

15) Z. Jiang, F. G. Sun, Q. Chen and X.-C. Zhang: Appl. Phys. Lett. 74 (1999) 1191

16) T. Hattori, K. Ohta, R. Rungsawang and K. Tukamoto: J. Phys. D 37 (2004) 770.

17) G. L. Eesley, M. D. Levenson and W. M. Tolles: IEEE J. Quantum Electron. 14 (1978) 45

18) T. Hattori, R. Rungsawang, K. Ohta and K. Tukamoto: Jpn. J. Appl. Phys. 41 (2002) 5198.

19) M. T. Reiten, S. A. Harmon and R. A. Cheville: J. Opt. Soc. Am. B 20 (2003) 2215

\section{Appendix}

Here we describe the optical configuration of phasesensitive electrooptic detection and derive eq. (1). The principle coordinate is chosen to be collinear with the laboratory frame so that the $x, y$ and $z$ axes align along the horizontal, vertical and propagation directions, respectively. Jones vectors and matrices represent the polarization state of the probe light as follows.

(1) The input probe beam is linearly polarized along the $x$ axis; namely, the polarization is in the (11) direction of the EO crystal. We represent the input field by a column vector

$$
\boldsymbol{E}_{\text {in }}=\left[\begin{array}{l}
1 \\
0
\end{array}\right] E_{0}
$$

(2) When the probe light passes through the EO crystal, the electric field components along the ( $(\overline{1} 10)$ and $(\overline{1} \overline{1} 0)$ directions are modulated with phase retardation $2 \theta$ due to the presence of a THz field along the $x$ axis. Assuming $\mathbf{R}(\psi)$ is the rotation matrix, which rotates the coordinates $x, y$ to the new coordinates $x^{\prime}, y^{\prime}$ by an angle $\psi$ about the $z$ axis, the transmission matrix of the crystal is

$$
\begin{aligned}
\mathbf{T}_{\mathrm{eo}} & =\mathbf{R}^{-1}\left(-\frac{\pi}{4}\right) \mathbf{T}_{\mathrm{eo}}^{(0)} \mathbf{R}\left(-\frac{\pi}{4}\right) \\
& =\left[\begin{array}{cc}
\cos \frac{\pi}{4} & \sin \frac{\pi}{4} \\
-\sin \frac{\pi}{4} & \cos \frac{\pi}{4}
\end{array}\right]\left[\begin{array}{cc}
e^{i \theta} & 0 \\
0 & e^{-i \theta}
\end{array}\right]\left[\begin{array}{rr}
\cos \frac{\pi}{4} & -\sin \frac{\pi}{4} \\
\sin \frac{\pi}{4} & \cos \frac{\pi}{4}
\end{array}\right] \\
& =\left[\begin{array}{cc}
\cos \theta & -i \sin \theta \\
-i \sin \theta & \cos \theta
\end{array}\right] .
\end{aligned}
$$

Here, $\mathbf{T}_{\mathrm{eo}}^{(0)}$ is the Jones matrix of the EO crystal in the frame along its optical axes.

(3) The transmitted beam passes through a quarter-wave plate whose fast axis is adjusted to be parallel to the $x$ axis. Therefore, its transmission matrix is

$$
\mathbf{T}_{\lambda / 4}=\left[\begin{array}{cc}
e^{i \pi / 4} & 0 \\
0 & e^{-i \pi / 4}
\end{array}\right] .
$$

(4) The probe light is then transmitted through a linear analyzer that is rotated by the angle $\delta$ from the $y$ axis. The Jones matrix of the analyzer becomes

$$
\begin{aligned}
\mathbf{T}_{\mathrm{a}} & =\mathbf{R}^{-1}(\delta) \mathbf{T}_{\mathrm{a}}^{(0)} \mathbf{R}(\delta) \\
& =\left[\begin{array}{cc}
\cos \delta & -\sin \delta \\
\sin \delta & \cos \delta
\end{array}\right]\left[\begin{array}{ll}
0 & 0 \\
0 & 1
\end{array}\right]\left[\begin{array}{cc}
\cos \delta & \sin \delta \\
-\sin \delta & \cos \delta
\end{array}\right] \\
& =\left[\begin{array}{cc}
\sin ^{2} \delta & -\sin \delta \cos \delta \\
-\sin \delta \cos \delta & \cos ^{2} \delta
\end{array}\right] .
\end{aligned}
$$

Therefore, the output probe field is described by

$$
\begin{aligned}
\boldsymbol{E} & =\left[\begin{array}{c}
E_{x} \\
E_{y}
\end{array}\right] \\
& =\mathbf{T}_{\mathrm{a}} \mathbf{T}_{\lambda / 4} \mathbf{T}_{\mathrm{eo}} \boldsymbol{E}_{\mathrm{in}} .
\end{aligned}
$$

The CCD camera detects the output intensity

$$
I=\left|E_{x}\right|^{2}+\left|E_{y}\right|^{2} .
$$

By substituting the transmission matrices and the vector into eq. (A.5), we obtain

$$
I=I_{0} \sin ^{2}(\delta+\theta)
$$

where $I_{0}=E_{0}^{2}$. If we consider the scattered light in the EO crystal, $\eta I_{0}$, and assume that $|\delta|$ and $|\theta|$ are small, eq. (A.7) becomes

$$
I=I_{0}\left[\eta+(\delta+\theta)^{2}\right] .
$$

Substituting $I_{\mathrm{b}}=I_{0}\left(\eta+\delta^{2}\right)$ in eq. (A.8), we get

$$
I-I_{\mathrm{b}}=I_{0}\left(2 \delta \theta+\theta^{2}\right) \text {. }
$$

Therefore, the phase retardation $\theta$ in the crystal is

$$
\theta=-\delta \pm \sqrt{\delta^{2}+\frac{I-I_{\mathrm{b}}}{I_{0}}} .
$$

Since $\theta$ should be small when $I-I_{\mathrm{b}}$ is small, we pick up only the positive sign in eq. (A-10), which leads to eq. (1). The THz field, $E_{\mathrm{THz}}$, is related to the phase retardation, $\theta$, by

$$
E_{\mathrm{THz}}=\frac{\lambda}{\pi n_{0}^{3} r_{41} L} \theta
$$

where $\lambda$ is the wavelength of probe light, $n_{0}$ is the refractive index of the EO crystal, $r_{41}$ is the element of the electrooptic tensor, and $L$ is the thickness of the crystal. 